\title{
On the Model of Prognosis of Proceeds of Industrial Enterprises
}

\author{
E.L. Pankratov ${ }^{1,2}$ \\ ${ }^{I}$ Nizhny Novgorod State University, 23 Gagarin Avenue, Nizhny Novgorod, 603950, Russia \\ ${ }^{2}$ Nizhny Novgorod State Technical University, 24 Minin Street, Nizhny Novgorod, 603950, Russia \\ elp2004@mail.ru
}

\begin{abstract}
In modern economic theory, scientific and technological progress considers as one of the relevant factors of long-term eco-nomic growth. The influence of scientific and technological progress on a particular sector of the economy appears in the creation of new products that have important competitive advantages over existing ones, or in the modification (modernization) of existing products. Often, new products based on new (innovative) technologies. However, technological superiority requires well-timed modernization of production and staff training, that is, significant financial and organizational investments. At the same time, the rejection of the transition to innovative technologies can lead to tangible losses in market positions or even to a complete cessa-tion of the organization. This model gives a possibility to make a prognosis of proceeds of enterprises with account changing of quantity of manufactured products, as well as various expenses (raw materials, transportation costs,...). An analytical approach for analyzing the influence of various parameters on the proceeds has been introduced.
\end{abstract}

\section{Keywords}

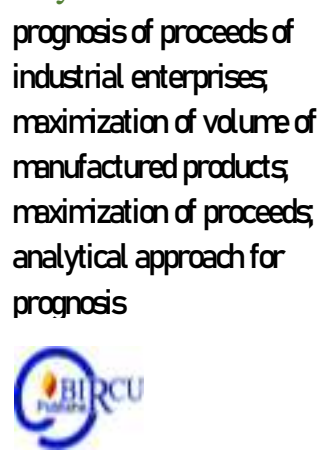

\section{Introduction}

In modern economic theory, scientific and technological progress considers as one of the relevant factors of long-term economic growth. The influence of scien-tific and technological progress on a particular sector of the economy appears in the creation of new products that have important competitive advantages over existing ones, or in the modification (modernization) of existing products. Often, new products based on new (innovative) technologies. However, technological superiority requires well-timed modernization of production and staff training, that is, significant financial and organizational investments. At the same time, the rejection of the transition to innovative technologies can lead to tangible losses in market positions or even to a complete cessation of the organization. Currently, one of the important examples of markets, which characterizing by the crowding out of some products by others that are more attractive from a technological point of view, is the market for information and telecommunication technologies (the market for data transmission services). For most managers of enterprises that are suppliers of products and investors, the issue of competitiveness of enterprises and their development prospects and its prognosis is relevant. The competitiveness of an enterprise is determined, first of all, by the quantity of manufactured products and sold, as well as the profit received. Lower costs and costs increase competitiveness. Profits and costs are affected by many factors that change over time. In this situation, it is of interest to formulate a methodolo-gy for predicting the competitiveness and optimization functioning of industrial enterprises. One of the main factors optimizing the functioning of industrial 
Konfrontasi Journal: Culture, Economy and Social Changes, 7 (4) December 2020, 253-263

ISSN: 1410-881X (Print), 2716-2095 (Online)

\section{E.L. Pankratov: On the Model of Prognosis of Proceeds of Industrial Enterprises}

DOI: https://doi.org/10.33258/konfrontasi2.v7i4.123

http://www.konfrontasi.net/index.php/konfrontasi2

enterprises is increasing of profits, as well as a decreasing of overhead costs (see, for example, Nathaphan \& Chunhachinda; Wu \& Hung; Prat; Yaghoubi, Yaghoubi, Locke, \& Gibb). In this situation, it is necessary to develop effective methods of innovative development and changing the market situation leading to price changes depending on the volume of products on the market (see, for ex-ample, Firdaus; Mertzanis; Pankratov, 2019; Nikoloski). In this paper we intro-duce a model for prognosis of proceeds of enterprises with regard to changes in the volume of production. An analytical method for analyzing the dependence of revenue on various parameters is also introduced.

\section{Review of Literatures}

\subsection{What this Prognosis}

It is obvious that entrepreneurship is an activity associated with investing and generating income. The funds are invested today, and the income will be received tomorrow. To assess the possible amount of income and the effectiveness of investments, it is necessary to determine not only the sequence of actions and calculate their expected result, but also the future state of the enterprise and the external environment, including the conditions for selling products, the behavior of competitors. Possible structure of assets and sources of their financing, etc. And without these estimates, investment performance calculations are unlikely to meet the minimum reliability requirements. Determination of the future state of the enterprise and its environment on the basis of the prevailing trends is forecasting. Whether we realize it or not. But when making any planned or unplanned decisions. Assessment of their possible consequences is a mandatory management action. And it is better that this action is carried out systematically and correctly as far as the available information allows. Evaluation of the consequences of decisions and actions for the enterprise, taking into account the current trends in changes in the external environment and the state of the enterprise, or forecasting differs from the planning of these actions and decisions only in that when planning we are guided primarily by the goal that must be realized, that is, based on the goal - we plan the sequence actions and the resources required for their implementation. When forecasting, the result or the possible degree of achievement of goals is the likely consequences of the decisions made or planned. In this sense, forecasting is a necessary component of planning and management. And planning success and. therefore, the management of the enterprise will be completely determined by the quality of predictive estimates of the consequences of decisions.

\subsection{Main Aims of Prognosis}

Forecasting the results of the enterprise and its financial condition is carried out in order to:

(i) Assessing the economic and financial prospects and the expected financial condition of the enterprise for the planned period, depending on the main possible options for its production and marketing activities and it's financing;

(ii) Formation on this basis of sound conclusions and recommendations regarding the choice of a rational strategy and tactics of actions of the top management of the enterprise.

Among the strategic and tactical decisions may be the production and sales program of the enterprise for the planned period, the planned structure of assets, including circulating assets, the principle scheme for financing the assets and activities of the enterprise for the planned period, the ability to implement one or another investment project, etc. That is, any decision on the use of financial resources and the consequences of the implementation of this decision for the financial condition of the enterprise can be subjected to a predictive 
assessment.

Taking into account the extremely unstable financial condition of a significant part of Russian enterprises. One of the tasks of financial forecasting can be to assess the opportunity. Basic conditions and terms of normalization of the state of the enterprise. That is, the possibilities and conditions for its financial recovery. In this sense, financial forecasting is a necessary element of crisis management.

\subsection{Financial Positioning in the Enterprise Management System}

Forecasting, as noted above is a necessary component of management and one of the main conditions for effective planning and this determines its importance in the enterprise management system. Any decision should be preceded by an analysis of the current situation and a forecast of the possible consequences of its adoption or not.

To make the analysis and forecasting procedures specific, to eliminate abstract assumptions like "Now, if only ..." and to bring the decision-making process into the boundaries of the adopted strategic priorities, it seems expedient to determine the normal parameters of the enterprise's activities, that is, the main indicators of its work that are considered the norm. In this case, the processes of analysis and forecasting will have the main content of comparing the actual (predicted) values of the parameters of the enterprise with normal ones, and the planning process will develop measures to bring the real state of the enterprise to normal.

\subsection{Methods for Prognosis}

The financial condition of an enterprise can be described quite correctly using three classical models: a balance of income and expenses, a balance of assets and liabilities, and a balance of receipts and payments. The same models allow you to assess the effectiveness and efficiency of the enterprise. Therefore, the methodological basis for forecasting the financial condition and performance of enterprises should be these three balances. The balance of income and expenses, which describes the results of the company's activities for the period, the balance of assets and liabilities, which creates the financial image of the enterprise and characterizes the structure of its assets and liabilities, and the balance of receipts and payments, which represents the movement of means of payment between the enterprise and its counterparties and gives a complete picture of the dynamics collection of receivables and financing of all operations of the enterprise for the period, together form the financial model of the enterprise. Therefore, forecasting the financial condition of the enterprise and the results of its activities is the process of creating options for the financial model of the enterprise. Taking into account any possible decisions on the formation of a production and sales program, on the implementation of investment projects, on the purchase of materials and raw materials, on determining the timing of the provision of commercial loans to consumers, on the formation of a payroll, on the purchase of three brooms, etc. etc.

The process of forming the financial model of an enterprise (and predicting its state) has the following sequence. The first is the balance of income and expenses. The projected (planned) results of the enterprise for the period and the initial state of assets and liabilities are the basis for designing the balance of assets and liabilities. The content of the two previous balances allows you to calculate (exactly calculate!) The flow of receipts and payments for the period.

Since the formation of three balances is an absolutely formalized procedure, the rules of which are determined by accounting standards, and the relationships between balances are just as formalized, the process of financial forecasting can be easily computerized. This allows you to quickly enough, in real time, make assessments of the consequences of any 
possible financial decisions.

The procedure for forecasting the financial condition and results of the enterprise includes, firstly. Preparation of initial information about the state of the enterprise and preparation of planning solutions, divided into six blocks. Block one - the initial state of the assets and liabilities of the enterprise, financial reporting data. Block two - the planned (projected) sales volume and conditions for the sale of products. This is information from the sales (marketing) service. Block three - planned investments and disinvestments in noncurrent assets. This information is prepared by the finance department on the basis of preliminary (design) planning decisions for the technical development of the enterprise. Block four - forecasted at the end of the period warehouse stocks of finished products and materials, work in progress, the amount of accounts receivable and other elements of current assets. Forecast estimates should be made by the finance department in consultation with the relevant departments of the enterprise. Block five - decisions on changing the authorized capital and payment of dividends. Block six - design solutions for financing the enterprise for the forecast period, including obtaining and returning long-term and short-term loans, changes in the value of commercial payables, balances of wages and payments to the budget and off-budget funds. In addition, for modeling, it is necessary to use a computerized unit for calculating taxes or enter information on the amount of payments calculated by other methods to be paid to the budget and extra-budgetary funds in during the forecast period. The second step is to structure the initial information in a certain way, that is, to enter it into the appropriate formats (tables). Further, on the basis of this structured information, a financial model of the enterprise and forecast balances of income and expenses, assets and liabilities, receipts and payments are created. The resulting balances are the basis for making a decision.

\subsection{Forecasting Period, Forecast Options}

The forecasting period can be fundamentally any: from a month to fifty years. His choice is determined. First, the goals of forecasting. That is, the nature of the decisions which are to be accepted using predictive estimates, and secondly, the reliability of the initial information. Obviously, it makes no sense to make predictive calculations when the error of some data, for example, sales volume exceeds 15 - 20\%. Such a forecast makes little sense, since decisions, the consequences of which have a probability of implementation $+20 \%$, can be made without laborious forecast calculations. In the current conditions of Russia, forecast calculations can give a completely correct result when choosing a forecasting period from several days to $2-2.5$ years. This choice is due to the fact what. On the one hand, a shortterm forecast is needed to assess the near-term prospects: on the other hand, to rationalize the choice and assess the strategy and tactics of action, the top management of the enterprise should assess its prospects for at least 2 years. Since during this period investments in more or less effective investment projects pay off.

To assess the impact on the financial condition and performance of the enterprise of probable changes in the main factors (sales, costs, etc.), it is advisable to make forecast calculations according to several options with different initial data (production program, structure of production costs, investments, etc.). In practice, it is customary to most often assess the future in three ways: pessimistic, optimistic and realistic. This allows executives! Enterprises to be prepared for unexpected troubles and fortunately on occasion.

On the procedure for the formation of the initial data for forecasting, the main assumptions that are advisable when carrying out forecast calculations. We plan to talk about the calculation techniques and methods of interpreting the results in the next articles of this cycle. 


\section{Research Methods}

In this paper as the model of proceeds we used the following expression

$$
Q=V \cdot P-V \cdot R-V \cdot T-V \cdot S
$$

Here $V$ is the quantity of manufactured product; $P$ is the price of a unit of the product; $R$ is the price of raw materials, consumed per unit of production; $T$ is the transportation costs per unit of product; $S$ is the salary paid to employees per unit of production. The price of products may vary depending on its quantity. In this paper, we consider the simplest (linear) model of such a dependence $P(V)=A-B \cdot V$, where $A$ and $B$ are the parameters of the approximating function, taking into account the actual price change (see, for example, Pankratov, 2019). Taking this approximation into account, relation (1) takes the following form

$$
Q=V \cdot(A-B \cdot V)-V \cdot R-V \cdot T-V \cdot S .
$$

Proceeds $Q$ depends on parameters $R, T, S, A, B$ monotonically. The dependence of proceeds $Q$ on the volume of product $V$ can be non-monotonic. Extreme proceeds value can be defined as standard, i.e. from the condition of equality to zero of the corresponding partial derivative: $\partial Q / \partial V=0$ (see, for example, Korn \& Korn). With account relation ( $1 a$ ) equation $\partial$ $Q / \partial V=0$ could be written as

$$
\partial Q / \partial V=A-2 B \cdot V-R-T-S=0
$$

From the relation (2) one can obtain the extreme value of the volume of manufacturing product $V_{\text {extr }}$

$$
V_{\text {extr }}=(A-R-T-S) / 2 B \text {. }
$$

The dependencies of the industrial enterprise's proceeds, as well as the extreme values of the product, are shown in the figures below. Figure 1 shows typical dependences of the volume of proceeds $Q$ on the volume of products $V$ for different values of parameters $A$ and $B$. Increasing of number of curves corresponds to increasing of value of parameter $A$ (Fig. $1 a$ ) and $B$ (Fig. 1b). The dependences of the volume of proceeds on the volume of output for various values of the parameters $R, T, S$ are similar to those shown in Fig. $1 a$.

\section{Results and Discussion}

It follows from these figures that the dependencies of the amount of proceeds on the volume of product for various values of parameters can be both monotonic and nonmonotonic with a pronounced extreme (in this case, maximum) value determined by relation (3). Fig. 2 shows the dependences of the proceeds volume on the parameters $R, T, S, A$ and $B$. All dependencies are straight lines with different angular coefficients. Depending on the values of the parameters, the predicted profit can be either positive or negative, which corresponds to the loss of the enterprise. 


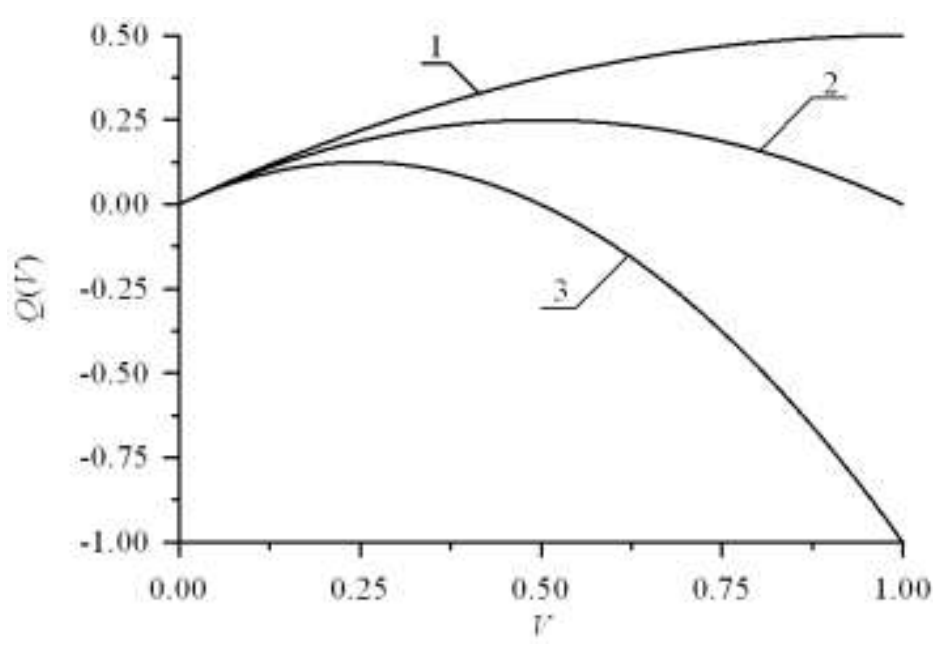

Figure 1a. The dependence of proceeds $Q$ on the volume of products $V$ for different values of the parameters $R, T, S, A$ (all dependencies are qualitatively similar to each other). An increase in the number of curves corresponds to an increase in the value of parameter $B$

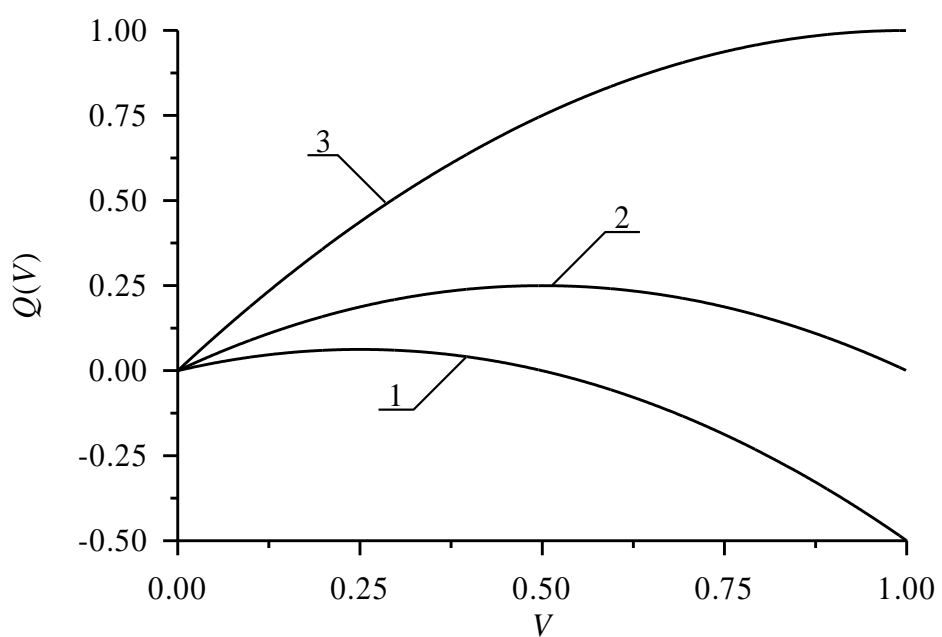

Figure $1 b$. Dependence of proceeds $Q$ on the volume of products $V$ for various values of the parameters $R, T, S, B$ (all dependences are qualitatively similar to each other). An increase in the number of curves corresponds to an increase in the value of the parameter $A$ 


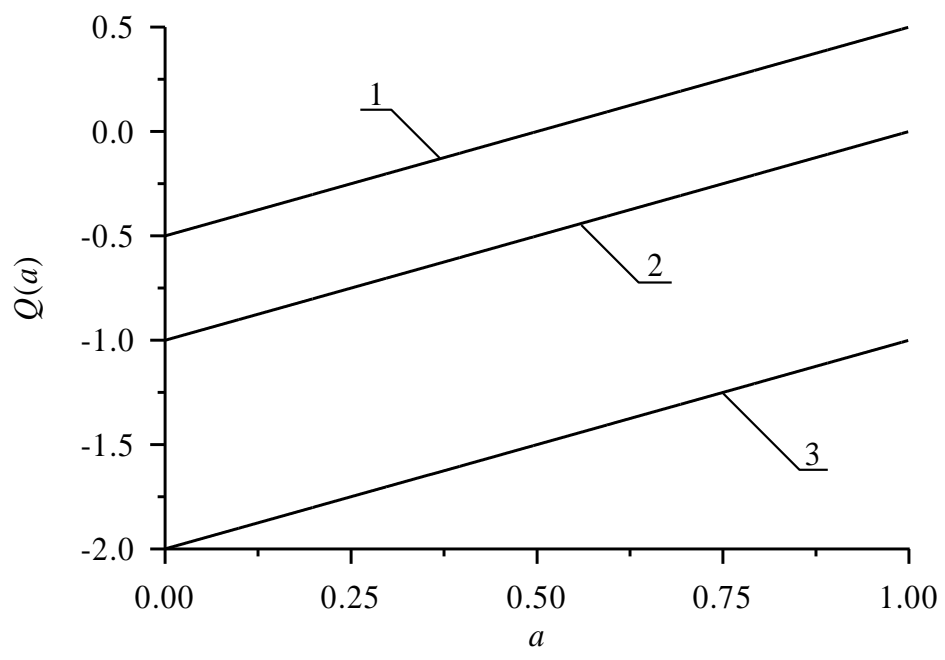

Figure 2a. The dependence of the proceeds $Q$ on the value of the parameter A for different values of the parameters $R, T, S, B$ (all dependences are qualitatively similar to each other). An increasing in the number of curves corresponds to increasing of value of the parameter $V$

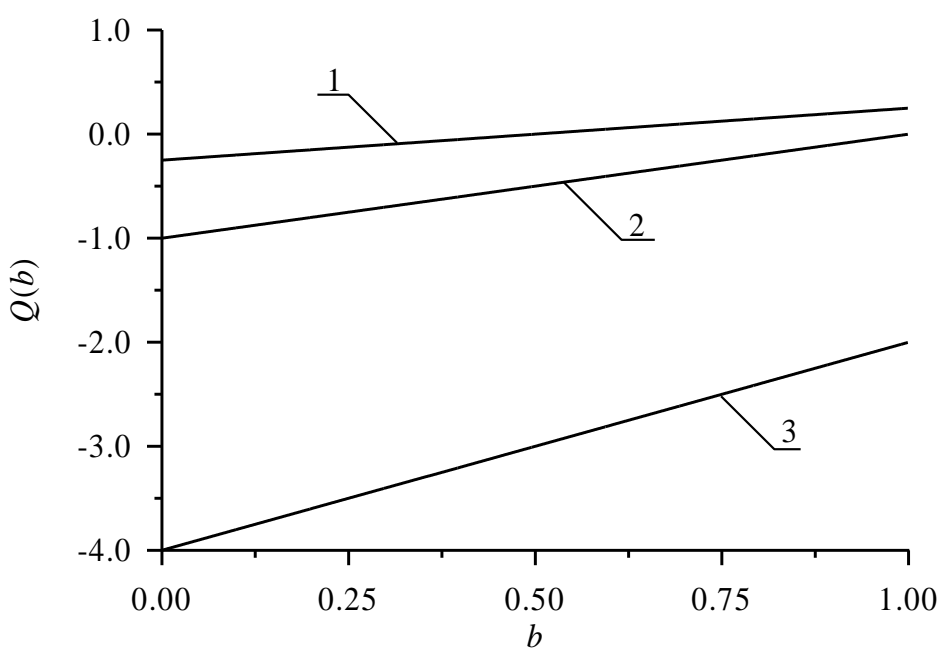

Figure $2 \boldsymbol{b}$. The dependence of proceeds $Q$ on the value of the parameter $B$ for different values of the parameters $R, T, S, A$ (all dependencies are qualitatively similar to each other). Increasing in the number of curves corresponds to increasing in the value of the parameter $V$

Figs. 3-5 show dependences of the maximum volume of manufactured products on a value of considered parameters for different values of other parameters. These dependences obtained are usually linear. Some of them are hyperbolic. 


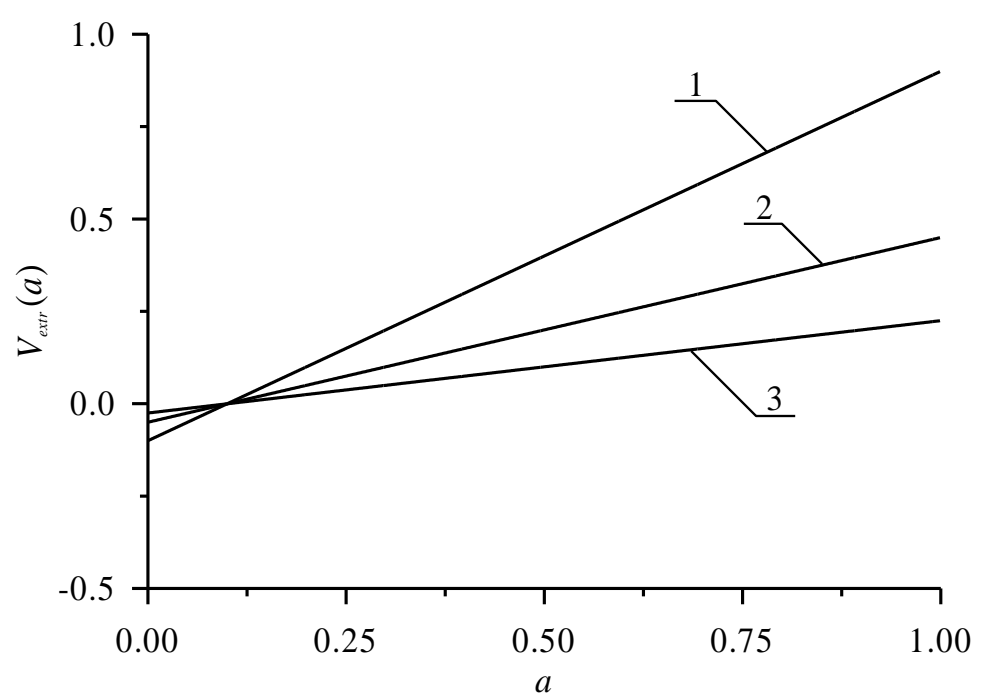

Figure 3a. Dependencies of the maximum volume of manufactured products on the values of parameter A. Increasing of number of curves corresponds to increasing of value of parameter $B$

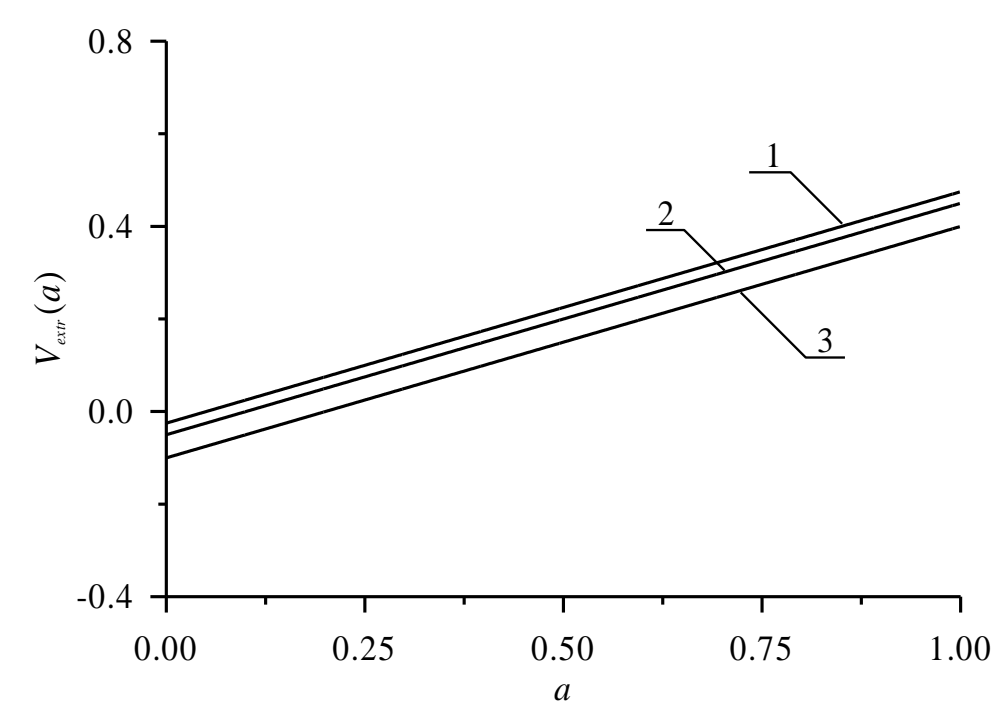

Figure 3b. Dependencies of the maximum volume of products on the values of parameters $A$. Increasing of number of curves corresponds to increasing of values of the parameters $A$ 


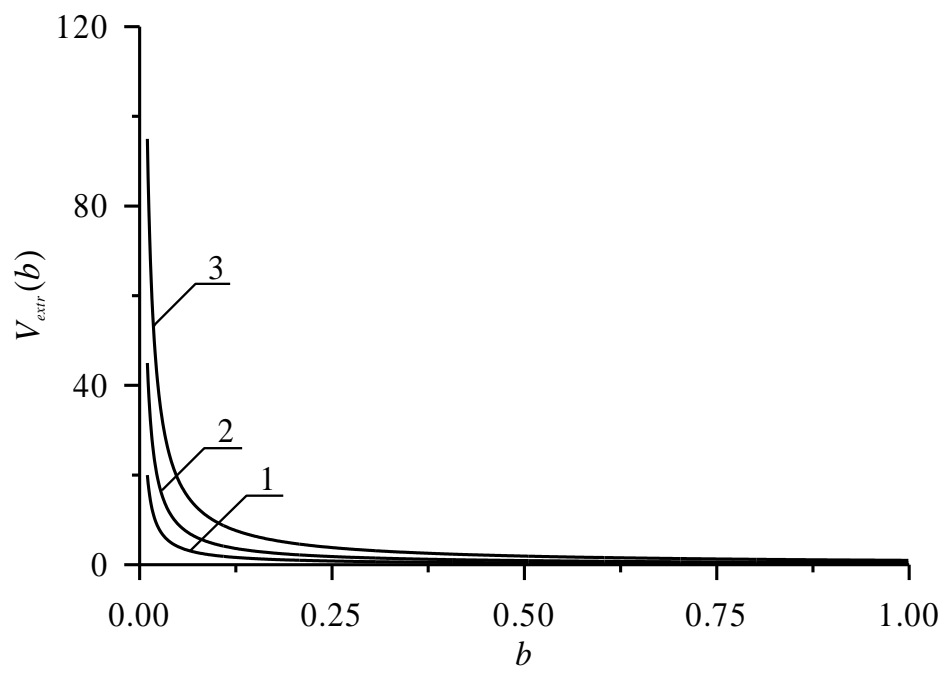

Figure 4a. Dependencies of the maximum volume of products on the values of parameter $B$. Increasing of number of curves corresponds to increasing of value of parameter $A$

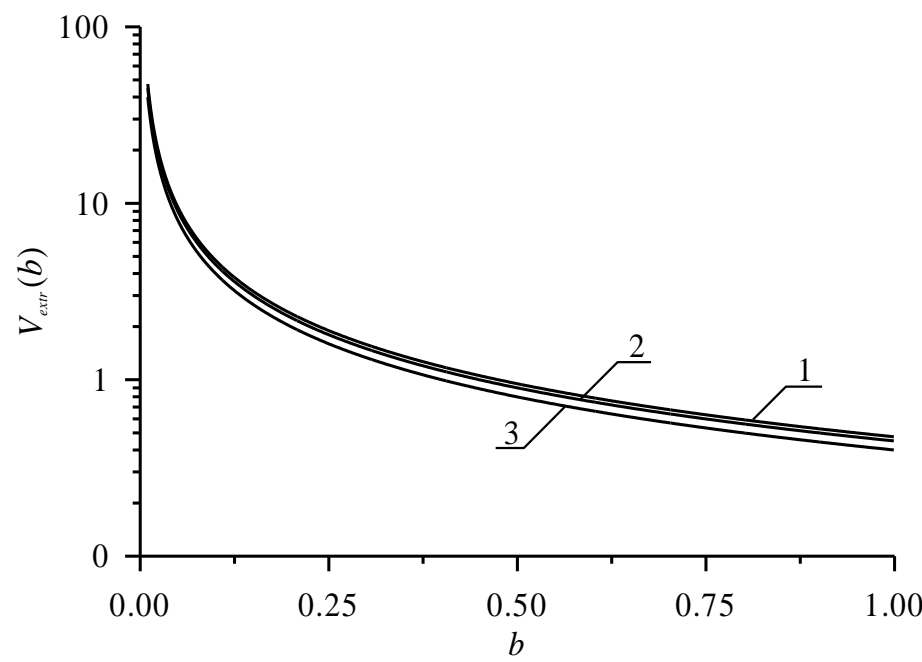

Figure 4b. Dependencies of maximum product on value of parameter B. Increasing of the number of curves corresponds to increasing of the values of the parameters $R, T, S$ 


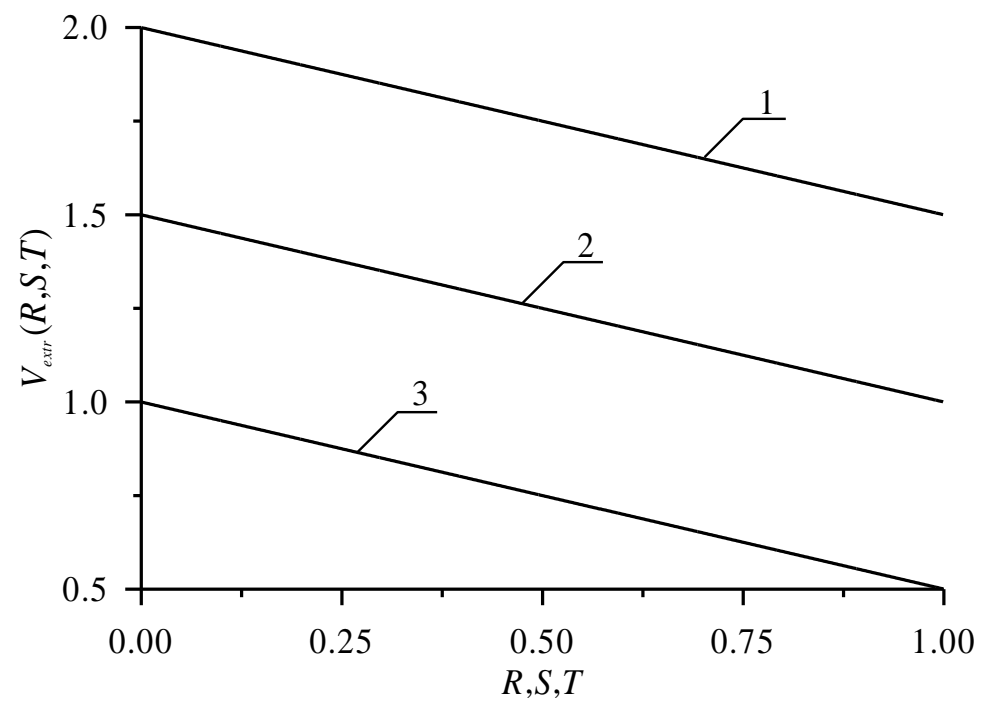

Figure 5a. Dependencies of the maximum volume of products on the values of the parameters $R, T, S$. Increasing of number of curves corresponds to increasing of value of parameter $A$

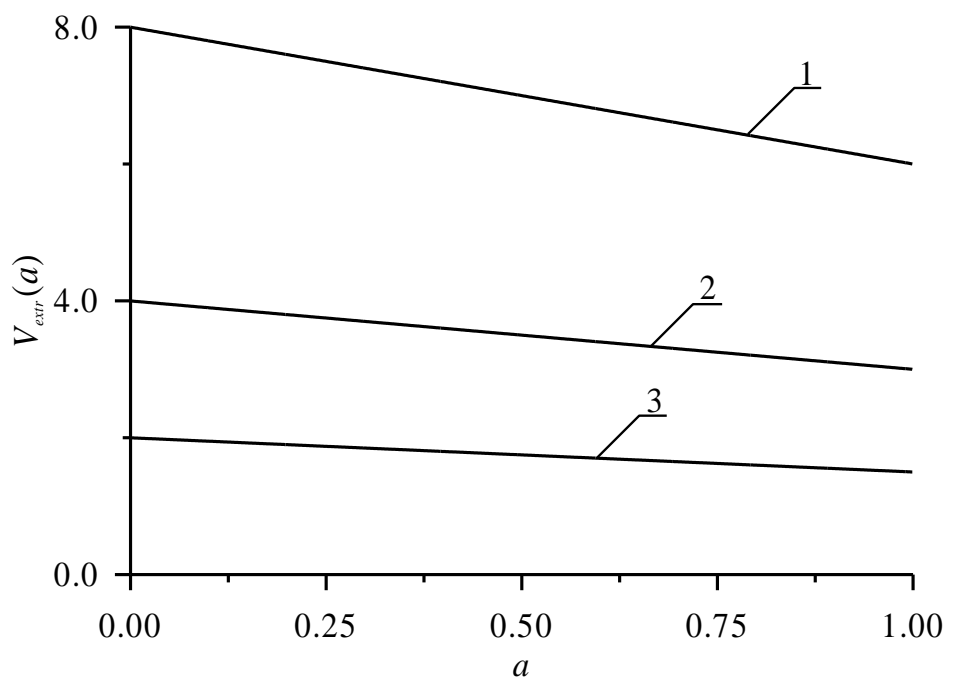

Figure $5 \boldsymbol{b}$. Dependencies of the maximum volume of products on the values of parameters $R$, $T$, $S$. Increasing of number of curves corresponds to increasing of values of the parameter $B$

\section{Conclusion}

This paper presents a model for prognosis of proceeds of industrial enterpris-es. This model gives a possibility to make a prognosis of proceeds of enterprises with account changing of quantity of manufactured products, as well as various expenses (raw materials, transportation costs, ...). An analytical approach for analyzing the influence of various parameters on the proceeds has been intro-duced. Based on this approach we analyzed dependences of proceeds of industri-al enterprises on different parameters. 


\section{References}

Firdaus, Q. (2019). A Philosophical Reflection in Controlling the Property and Selfownership. Konfrontasi Journal: Culture, Economy and Social Changes, 6 (2), 1-7.

Korn, G. \& Korn, T. (1968). Mathematical Handbook for scientists and engineers. Definitions, theorems and formulas for reference and review. Second edition. New York: McGraw-Hill Book Company.

Mertzanis, Ch. (2017). Short selling regulation, return volatility and market volatility in the Athens Exchange. Studies in economics and finance, 34 (1), 82-104.

Nathaphan, S. \& Chunhachinda, P. (2010). Estimation risk modeling in optimal portfolio selection: an empirical study from emerging markets. Economics research international, Vol. 2010. Article ID 340181.

Nikoloski, A. (2020). Influence of public revenues on economic growth the case of the Republic of North Macedonia. Konfrontasi Journal: Culture, Economy and Social Changes, 7 (3), 191-204.

Pankratov, E.L. (2018). Nonlinear profit maximization with account changing of prices. International journal of modeling, simulation and applications, 2 (2), 9-17.

Pankratov E.L. (2019). Model for prognosis of economic growth: accounting of influence of the environment. Global economy journal, 19 (1), 1950007-1--1950007-2.

Prat, J. (2015). Dynamic contracts and learning by doing. Mathematics and financial economics, 9 (3), 169-193.

Wu, Ch.W., \& Hung, W.Zh. (2010). Real national income average growth rate: a novel economic growth and social fair evaluation index. Economics research international, Vol. 2010. Article ID 678927.

Yaghoubi, R., Yaghoubi, M., Locke, St. \& Gibb, J. (2016). Mergers and acquisitions: a review (part 2). Studies in economics and finance, 33 (3), 437-464. 